onset. CT/MRI imaging; urine 5HIAA and plasma chromagranin A (CgA) and toxicities were recorded.

Results 9 (24\%) withdrew before 3 months because of toxicity, progressive disease or death. On intention to treat analysis: $1(3 \%)$ had complete response; 1 (3\%) partial response; 26 (70\%) had at least 3 months of stable disease. The median TTP was 14 months. Median 5HIAA fell from 54 to 29 umol/24h at 6 months (NS) and CgA from 138 to $121 \mathrm{pmol} / 1$ at 6 months (NS). 38\% had WHO grade 1-2 haematological toxicity and $19 \%$ grade $3-4$. The only other grade 3-4 toxicity was depression in 1 patient and $22 \%$ had grade 1-2 depression. Other grade 1-2 toxicities $>10 \%$ included flu-like symptoms $24 \%$, fatigue $16 \%$, hypothyroidism $11 \%$, dry skin $14 \%$.

Conclusion Although there is toxicity which affects management in up to $1 / 3$ rd of patients the remaining patients tolerated therapy well. IFN $\alpha$ demonstrated efficacy in at least inducing or maintaining stable disease in most patients $(76 \%)$. The median TTP is at least similar to other molecular targeted therapies. Those patients who were going to be intolerant or progress usually did so within the first 3 months of treatment. It would be appropriate to perform prospective randomised studies utilising IFN $\alpha$ and also better assess quality of life.

Disclosure of Interest None Declared.

\section{PWE-161 SESSILE SERRATED ADENOMAS, UNDER-RECOGNISED ENDOSCOPICALLY AND UNDER DIAGNOSED PATHOLOGICALLY}

doi:10.1136/gutjnl-2013-304907.449

1." H Rafferty, ${ }^{2} \mathrm{P}$ Gill, ${ }^{1} \mathrm{H}$ Davis, ${ }^{3} \mathrm{~A}$ Bailey, ${ }^{3} \mathrm{~J}$ East, ${ }^{2} \mathrm{R}$ Chetty, ${ }^{1,3}$ S Leedham. ${ }^{1}$ Molecular and Population Genetics, University of Oxford; ${ }^{2}$ Histopathology; ${ }^{3}$ Translational Gastroenterology Unit, John Radcliffe Hospital, Oxford, UK

Introduction The serrated pathway of colorectal carcinogenesis is a distinct and important pathway leading to CpG island methylated phenotype (CIMP) carcinomas. These lesions are over-represented in interval cancers and may explain the failure to prevent right-sided cancer with colonoscopy. Hyperplastic polyps (HPs) in the left colon rarely transform whereas proximal serrated adenomas (SSAs) have definite malignant potential and are notoriously difficult to detect endoscopically. There is still uncertainty surrounding the diagnostic criteria and management implications of SSA's, however as pre-malignant lesions it is vital to find, resect and diagnose them. We assessed SSA diagnosis over 4 years in a teaching hospital, and assessed the (epi)genetic mutation burden and expression profile of rectal HPs versus proximal SSAs to see if identifiable molecular differences contribute to their contrasting malignant potential. Methods We searched the pathology archives from 2009 to 2012 for the diagnosis of serrated adenomas. Cases were reviewed by 2 GI pathologists. Colonoscopic follow up for each case was assessed and compared with new American guidelines ${ }^{1} .5$ distal HP and proximal SSA samples were obtained endoscopically, individual crypts were dissected and morphogen gene expression analysed. Obtained DNA was assessed for BRAF and KRAS mutation and CIMP status by methylight.

Results There were no serrated lesions diagnosed in 2009 - this was an unrecognised entity in our hospital at the time. Of 486 'hyperplastic' polyps diagnosed in 2009, 60 proximal lesions were reassessed and 19 were confirmed as SSAs. In 2010, 40 cases of SSA were diagnosed, rising to 84 in 2011 and 130 in 2012. Follow up of SSAs was appropriate in the majority of cases but lesions aberrantly denoted as hyperplastic in 2009 did not all have follow up arranged at the time. Molecular assessment showed a significant difference in (epi)mutation burden and morphogen gene expression between distal hyperplastic polyps and proximal SSA's.

Conclusion In our hospital there was no distinction made between hyperplastic and serrated lesions prior to 2010. A 3-fold increase in SSA diagnosis in the following 3 years reflects improved endoscopic detection and pathologist recognition of these lesions. The molecular difference between distal HPs and SSAs underpins the proximal predilection of CIMP cancers, and may reflect underlying differences in colonic regional microenvironment, microbiome or morphogen balance. Improved recognition of subtle endoscopic and morphological characteristics of SSAs by gastroenterologists and pathologists will improve colonoscopic surveillance.

Disclosure of Interest None Declared.

\section{REFERENCE}

1. Rex DK et al. Am J Gastroenterol 2012; 107(9):1315-29.

\section{PWE-162 CONCURRENT COMPUTERISED TOMOGRAPHY CAN OPTIMISE THE DETECTION OF CANCER IN PATIENTS PRESENTING WITH UNEXPLAINED ANAEMIA}

doi:10.1136/gutjnl-2013-304907.450

1."J Bains, 'S Mansukhani, 'S Coates, '2D J Pinato, 'M A Mendall. 'Gastroenterology, Croydon University Hospital, Iondon; ${ }^{2}$ ICTEM, Imperial, London, UK

Introduction Active investigation for gastrointestinal (GI) cancers is often triggered by "alarm symptoms"; features in the clinical presentation that may predict malignancy and warrant urgent referral. Unexplained anaemia (UA) is a highly prevalent presentation. The BSG guidelines recommend only upper GI endoscopy (OGD) and colonoscopy (COL). We investigated the additional diagnostic value of concurrent contrast enhanced computerised tomography of the chest, abdomen and pelvis (CT) in the investigation of patients (pts) aged $>50$ referred to the urgent suspected cancer (USC) pathway for GI malignancies. We evaluated its accuracy in detecting upper GI, lower GI and extraluminal malignancies in a cohort of consecutive pts presenting with and without UA.

Methods We retrospectively analysed characteristics and outcomes of 350 consecutive GI USC referrals (07/2010-07/2012): 200 (Group A) presented with UA and were investigated with OGD (178, 89\%), COL $(70,39 \%)$ and CT $(157,78 \%$, with $138,87 \%$ aged $>50$ years). The diagnostic outcomes were compared with a second group of 150 pts (Group B) referred with alarm symptoms (unintentional weight loss, abdominal pain, progressive dysphagia) who underwent OGD (91, 60\%), COL $(32,21 \%)$ and CT $(139,93 \%$, with $121,89 \%$ aged $>50$ years)

Results Group A had a mean age of 70 years (range 22-96), 51\% males, mean haemoglobin ( $\mathrm{Hb})$ of $10.2(5-13.8) \mathrm{g} / \mathrm{dL}$. Pts in Group $\mathrm{B}$ had a mean age of 67 years (range $20-92$ ), $60 \%$ males, mean $\mathrm{Hb}$ 13.9 (11.5-17.5) g/dL. Malignancy was diagnosed in 38 (19\%) Group $A$ and $17(12 \%)$ Group B patients $(p=0.07)$. The proportion of malignant cases diagnosed endoscopically was not different across the studied groups $(4 / 176,2 \%$ and $7 / 86,8 \%$ for OGD and COL in Group A; 5/91, 6\% and 3/32, 9\% in Group B, p = 0.3). Conversely, the rate of incident cancers identified by CT favoured Group A (33/157, $21 \%$ vs. $10 / 139,7 \%, p<0.001)$, where $71 \%$ of the incident cancers were extraluminal and diagnosed in pts $>50(29 / 33,87 \%)$.

Conclusion Concurrent CT can optimise the detection of malignancy in pts over the age of 50 referred under the GI USC pathway, with subjects presenting with unexplained anaemia achieving the greatest diagnostic benefit. We therefore propose that CT should be incorporated within the routine investigation pathway of anaemia in the over 50 s.

Disclosure of Interest None Declared.

\section{PWE-163 CHEMR23 AND BLT1 RECEPTOR EXPRESSION IN COLORECTAL CANCER}

doi:10.1136/gutjnl-2013-304907.451

1," J M Hutchinson, ${ }^{1} \mathrm{M}$ Volpato, ${ }^{2} \mathrm{P}$ Loadman, ${ }^{3} \mathrm{~A}$ Nicolaou, ${ }^{1} \mathrm{M}$ Hull. ${ }^{1}$ Molecular Gastroenterology, Leeds Insitute of Molecular Medicine, Leeds; '2Institute of Cancer Therapeutics, ${ }^{3}$ Bradford School of Pharmacy, University of Bradford, Bradford, UK 